\section{Additions to the flora of Sirumalai hills, Eastern Ghats, India}

\section{R. Vijaya Sankar ${ }^{1}$, R. Kottaimuthu ${ }^{2} \&$ K. Ravikumar ${ }^{3}$ \\ ${ }^{1,3}$ Foundation for Revitalisation of Local Health Traditions (FRLHT), Bangalore, Karnataka 560064, India \\ ${ }^{2}$ Ashoka Trust for Research in Ecology and the Environment \\ (ATREE), Bangalore, Karnataka 560024, India \\ Email: ${ }^{3}$ k.ravikumar@frlht.org (Corresponding author)}

The Sirumalai hills, situated in the southern most part of the Eastern Ghats in Tamil Nadu are known for their rich biodiversity for their close location to the Kodaikanal hills of the Western Ghats. They lie in Dindigul district, between $10^{\circ} 00^{\prime}-10^{\circ} 30^{\prime} \mathrm{N} \& 77^{\circ} 33^{\prime}-78^{\circ} 15^{\prime} \mathrm{E}$ and at altitudes ranging from 400 to $1650 \mathrm{~m}$. The lower hill ranges consist of highly disturbed scrub forests while tropical dry deciduous forests occupy the major portion of middle hill ranges. Semi-evergreen forests occur in the higher elevations and along valleys. Woodland savannahs are found along slopes. Bestowed with several endemic and medicinal plants, these hill ranges are facing severe threats owing to the growing anthropogenic disturbances and increasing coffee estates, rubber plantations, orchards and cultivation of several vegetable crops.

In spite of their rich plant wealth, the Sirumalai hills, have not been completely studied floristically in the recent times except 'A Pocket Flora of the Sirumalai Hills, South India' (Pallithanam 2001). It enumerates 895 species belonging to 536 genera of higher plants (excluding exotics). However, this work was compiled by K.M. Matthew based on the collections made by J.M. Pallithanam during 1955-1960. Subsequent to this pioneering work, for nearly half-a-century, only a few collections (Karuppusamy et al. 2001; Kottaimuthu et al. $2008 \mathrm{a} \& \mathrm{~b}$ ) have been made in the recent past in these hills.

Date of online publication 26 July 2009

ISSN 0974-7907 (online) | 0974-7893 (print)

Editor: J.L. Ellis

Manuscript details:

Ms \# 02069

Received 26 September 2008

Final received 01 January 2009

Finally accepted 30 May 2009

Citation: Sankar,R.V., R. Kottaimuthu \& K. Ravikumar (2009). Additions to the flora of Sirumalai Hills, Eastern Ghats, India. Journal of Threatened Taxa 1(7): 379-381.

Copyright: (C) R. Vijaya Sankar, R. Kottaimuthu \& K. Ravikumar 2009. Creative Commons Attribution 3.0 Unported License. JoTT allows unrestricted use of this article in any medium for non-profit purposes, reproduction and distribution by providing adequate credit to the authors and the source of publication.

Acknowledgement: The authors are thankful to Shri D.K. Ved, Director and Dr. G.S. Goraya, Joint Director, FRLHT, Bangalore for facility and encouragement; Shri Nihar Ranjan, District Forest Officer, Dindigul division for permission to conduct botanical surveys; and the Ministry of Environment \& Forests, Govt. of India for finalcial support under the 'Centre of Excellence' project. The second author is thankful to Dr. Ravi Chellam, Director and Dr. R. Ganesan, Scientist, ATREE, Bangalore for facility and encouragement and the Department of Biotechnology, Govt. of India for financial support.

OPEN AGGESS | FREE DOWNLOAD
Botanical surveys were conducted in 2007 , to enrich the collection of medicinal plants for FRLH (Herbarium of FRLHT, Bangalore), as part of 'Center of Excellence' project. This has resulted in the collection of 224 plant species under 237 field numbers. During the period of 2005-2008, botanical surveys were also conducted by ATREE for project on 'Mapping and quantitative assessment of plant bio-resources' in this region that resulted in the collection of 150 more species. Of these, 85 taxa are not reported earlier from the region and therefore form additions to the flora of Sirumalai hills (Table 1). All the herbarium specimens are deposited at FRLH (acronym of FRLHT Herbarium, Bangalore).

These 85 plant species spread over to 50 families of flowering plants and majority of them are herbs (40 species) followed by 21 trees, 14 shrubs and 10 climbers. A few introduced species such as Maesopsis eminii Engl. (Rhamnaceae) - a fast growing timber tree commonly planted in the coffee estates, Pimenta officinalis Lindl. (Myrtaceae) - the 'all spice' plant in addition to a few cereals, pulses and other vegetable crops have also been collected.

These studies indicate that several floristically rich areas in the State still remain botanically under-explored. Similar exercises in under/un-explored biodiversity rich areas would not only update the flora of the region but also bring out new distributional records to light.

\section{References}

Karuppusamy, S., K.M. Rajasekaran \& S. Karmegam (2001). Endemic flora of Sirumalai hills (Eastern Ghats), south India. Journal of Economic and Taxonomic Botany 25(2): 367-373.

Kottaimuthu, R., R. Ganesan, K. Natarajan, J. Brabhu \& M. Vimala (2008). Additions to the flora of Eastern Ghats, Tamil Nadu, India. Ethnobotanical Leaflets 12: 299-304.

Kottaimuthu, R., R. Ganesan, V. Ganesan \& V.M. Sundaram (2008). Enumeration of Orchids of Sirumalai hills (Eastern Ghats), Tamil Nadu, India. Ethnobotanical Leaflets 12: 506-512.

Pallithanam, J.M. (2001). In: Matthew, K.M. A pocket Flora of the Sirumalai Hills, South India. The Rapinat Herbarium, St.Joseph's College, Tiruchirapalli, 360pp. 
Table 1. List of plant species added to the flora of Sirumalai hills

\begin{tabular}{|c|c|c|c|c|c|}
\hline S.No & Botanical Name & Family & Habit & Collection No. & Phenology \\
\hline 1 & Adenia wightiana (Wight \& Arn.) Engl. & Passifloraceae & Climber & RKM 107508 & Flowers \\
\hline 2 & Alternanthera paronychioides A.St.Hil. & Amaranthaceae & Herb & KRK \& RVS 106232 & Flowers \& Fruits \\
\hline 3 & Amaranthus hybridus L. & Amaranthaceae & Herb & KRK \& RVS 106315 & Flowers \& Fruits \\
\hline 4 & Andrographis ovata (T.And. ex Bedd.) Benth. ex C.B. Clarke & Acanthaceae & Herb & RKM 107507 & Flowers \& Fruits \\
\hline 5 & Antidesma alexiteria $\mathrm{L}$. & Stylaginaceae & Tree & RKM 107523 & Flowers \\
\hline 6 & Asclepias curassavica L. & Asclepiadaceae & Herb & KRK \& RVS 106233 & Flowers \& Fruits \\
\hline 7 & Bergia ammannioides Roxb. ex Roth & Elatinaceae & Herb & RKM 107533 & Flowers \\
\hline 8 & Blumeopsis flava (DC.) Gagnep. & Asteraceae & Herb & KRK \& RVS 106281 & Flowers \& Fruits \\
\hline 9 & Capparis kollimalayana Viswanathan & Capparaceae & Liana & KRK \& RVS 106264 & Fruits \\
\hline 10 & Capsicum annuum L. & Solanaceae & Herb & KRK \& RVS 106305 & Fruits \\
\hline 11 & Casearia zeylanica Thwaites & Flacourtiaceae & Tree & RKM 107513 & Fruits \\
\hline 12 & Catunaregam torulosa (Dennst.) Tirveng. & Rubiaceae & Tree & KRK \& RVS 106214 & Flowers \& Fruits \\
\hline 13 & Chamaecrista kleinii (Wight \& Arn.) K.M.Matthew & Caesalpiniaceae & Herb & KRK \& RVS 106260 & Flowers \& Fruits \\
\hline 14 & Chlorophytum laxum R.Br. & Anthericaceae & Herb & KRK \& RVS 106331 & Flowers \& Fruits \\
\hline 15 & Cissus pallida Planch. & Vitaceae & Climber & RKM 106393 & Flowers \\
\hline 16 & Citrus medica L. & Rutaceae & Tree & KRK \& RVS 106344 & Fruits \\
\hline 17 & Cleome aspera J.Koenig ex DC. & Cleomaceae & Herb & RKM 107520 & Fruits \\
\hline 18 & Corchorus tridens $\mathrm{L}$. & Tiliaceae & Herb & RKM 107509 & Flowers \& Fruits \\
\hline 19 & Cordia obliqua Willd. var. tomentosa (Wall.) Kazmi & Cordiaceae & Tree & KRK \& RVS 106268 & Fruits \\
\hline 20 & Cryptolepis buchananii Roem. \& Schult. & Asclepiadaceae & Climber & KRK \& RVS 106335 & Flowers \& Fruits \\
\hline 21 & Cynoglossum zeylanicum (Vahl ex Hornem.) Thunb. ex Lehm. & Boraginaceae & Herb & KRK \& RVS 106329 & Flowers \& Fruits \\
\hline 22 & Daphniphyllum neilgherrense (Wight) K.Rosenthal & Daphniphyllaceae & Tree & RKM 106388 & Flowers \\
\hline 23 & Datura stramonium L. & Solanaceae & Herb & KRK \& RVS 106230 & Flowers \& Fruits \\
\hline 24 & Dendrobium heterocarpum Wall. ex Lindl. & Orchidaceae & Epiphytic herb & KRK \& RVS 106359 & Flowers \\
\hline 25 & Desmodium biarticulatum (L.) F.Muell. & Fabaceae & Herb & RKM 107530 & Flowers \& Fruits \\
\hline 26 & Diospyros ferrea (Willd.) Bakh. var. neilgherrensis (Wight) Bakh. & Ebenaceae & Tree & KRK \& RVS 106271 & Fruits \\
\hline 27 & Diospyros ovalifolia Wight & Ebenaceae & Tree & KRK \& RVS 106270 & Fruits \\
\hline 28 & Dyschoriste madurensis (Burm.f.) Kuntze & Acanthaceae & Woody herb & RKM 107515 & Fruits \\
\hline 29 & Euphorbia thymifolia L. & Euphorbiaceae & Prostrate herb & KRK \& RVS 106262 & Flowers \& Fruits \\
\hline 30 & Gisekia pharnaceoides L. & Molluginaceae & Spreading herb & RKM 107512 & Flowers \& Fruits \\
\hline 31 & Glochidion tomentosum Dalz. & Euphorbiaceae & Shrub & KRK \& RVS 106353 & Flowers \& Fruits \\
\hline 32 & Grewia tenax (Forssk.) Fiori & Tiliaceae & Shrub & RKM 106373 & Flowers \\
\hline 33 & Heliotropium bracteatum R.Br. & Boraginaceae & Herb & RKM 107516 & Flowers \& Fruits \\
\hline 34 & Heliotropium ovalifolium Forssk. & Boraginaceae & Herb & RKM 106390 & Flowers \& Fruits \\
\hline 35 & Heliotropium scabrum Retz. & Boraginaceae & Spreading herb & RKM 107517 & Flowers \& Fruits \\
\hline 36 & $\begin{array}{l}\text { Hemidesmus indicus (L.) R.Br. var. pubescens } \\
\text { (Wight \& Arn.) Hook.f. }\end{array}$ & Periplocaceae & Twiner & KRK \& RVS 106288 & Flowers \\
\hline 37 & Hydrocotyle javanica Thunb. & Apiaceae & Herb & KRK \& RVS 106278 & Flowers \\
\hline 38 & Indigofera parviflora Heyne ex Wight \& Arn. & Fabaceae & Herb & RKM 107510 & Flowers \\
\hline 39 & Isodon walkeri (Arn.) H.Hara & Lamiaceae & Herb & RKM 106372 & Flowers \& Fruits \\
\hline 40 & Justicia gendarussa Burm.f. & Acanthaceae & Shrub & RKM 106377 & Flowers \\
\hline 41 & Leea indica (Burm.f.) Merr. & Leeaceae & Shrub & RKM 107524 & Flowers \\
\hline 42 & Lepidium ruderale $\mathrm{L}$. & Brassicaceae & Herb & KRK \& RVS 106330 & Flowers \& Fruits \\
\hline 43 & Limnophila chinensis (Osbeck) Merr. & Scrophulariaceae & Herb & RKM 106383 & Flowers \& Fruits \\
\hline 44 & Lindernia ciliata (Colsm.) Pennell & Scrophulariaceae & Herb & RKM 107537 & Flowers \& Fruits \\
\hline 45 & Lindernia oppositifolia (Retz.) Mukerjee & Scrophulariaceae & Herb & RKM 107536 & Flowers \& Fruits \\
\hline 46 & Madhuca indica J.F.Gmel. & Sapotaceae & Tree & RKM 106389 & Fruits \\
\hline 47 & Manilkara roxburghiana (Wight) Dubard & Sapotaceae & Small tree & RKM 107535 & Fruits \\
\hline 48 & Mitracarpus villosus (Sw.) DC. & Rubiaceae & Herb & KRK \& RVS 106349 & Flowers \& Fruits \\
\hline 49 & Momordica charantia L. var. muricata (Willd.) Chakrav. & Cucurbitaceae & Climber & KRK \& RVS 106246 & Flowers \& Fruits \\
\hline 50 & Murdannia spirata (L.) G.Bruckn. & Commelinaceae & Herb & RKM 107526 & Flowers \& Fruits \\
\hline 51 & Mussaenda hirsutissima (Hook.f.) Hutchinson ex Gamble & Rubiaceae & Shrub & RKM 107532 & Flowers \& Fruits \\
\hline 52 & Oberonia brunoniana Wight & Orchidaceae & Epiphytic herb & KRK \& RVS 106360 & Fruits \\
\hline 53 & Ocimum gratissimum $\mathrm{L}$. & Lamiaceae & Undershrub & KRK \& RVS 106228 & Flowers \& Fruits \\
\hline 54 & Olax scandens Roxb. & Olacaceae & Straggler & RKM 107519 & Flowers \\
\hline 55 & Olea paniculata R.Br. & Oleaceae & Tree & RKM 106376 & Fruits \\
\hline 56 & Osyris wightiana J.Graham & Santalaceae & Shrub & RKM 106374 & Flowers \\
\hline 57 & $\begin{array}{l}\text { Oxyceros rugulosus (Thwaites) Tirveng. var. speciosus } \\
\text { (Bedd.) Kumari }\end{array}$ & Rubiaceae & Straggler & RKM 106371 & Flowers \\
\hline 58 & Pavetta indica L. var. montana Thwaites ex Hook.f. & Rubiaceae & Shrub & KRK \& RVS 106311 & Flowers \\
\hline 59 & Peliosanthes teta Andr. subsp. humilis (Andr.) Jessop & Haemodoraceae & Herb & KRK \& RVS 106324 & Fruits \\
\hline 60 & Pentatropis capensis (L.f.) Bullock & Asclepiadaceae & Twiner & RKM 107518 & Flowers \\
\hline 61 & Phyllanthus indofischeri Bennet & Euphorbiaceae & Tree & KRK \& RVS 106300 & Vegetative \\
\hline 62 & Phyllanthus kozhikodianus Sivar. \& Manilal & Euphorbiaceae & Herb & KRK \& RVS 106294 & Flowers \& Fruits \\
\hline 63 & Phyllanthus polyphyllus Willd. & Euphorbiaceae & Tree & RKM 107503 & Flowers \& Fruits \\
\hline 64 & Pittosporum tetraspermum Wight \& Arn. & Pittosporaceae & Tree & RKM 106382 & Flowers \\
\hline 65 & Pleurostylia opposita (Wall.) Alston & Celastraceae & Tree & RKM 106378 & Flowers \\
\hline 66 & Polyalthia korintii (Dunal) Thwaites & Annonaceae & Shrub & RKM 106369 & Flowers \\
\hline 67 & $\begin{array}{l}\text { Canthium dicoccum (Gaertn.) Teijsm. \& Binn. var. } \\
\text { umbellata (Wight) Sant. \& Merch. }\end{array}$ & Rubiaceae & Tree & RKM 106398 & Fruits \\
\hline 68 & Schrebera swietenioides Roxb. & Oleaceae & Tree & RKM 107514 & Vegetative \\
\hline 69 & Scilla hyacinthina (Roth) J.F.Macbr. & Liliaceae & Herb & RKM 107527 & Flowers \& Fruits \\
\hline 70 & Scolopia crenata (Wight \& Arn.) Clos & Flacourtiaceae & Tree & RKM 106387 & Flowers \\
\hline 71 & Scoparia dulcis L. & Scrophulariaceae & Herb & RKM 107529 & Flowers \& Fruits \\
\hline 72 & Scutellaria violacea B.Heyne ex Benth. & Lamiaceae & Herb & KRK \& RVS 106234 & Flowers \& Fruits \\
\hline 73 & Semecarpus anacardium L.f. & Anacardiaceae & Tree & RKM 107522 & Flowers (Male) \\
\hline 74 & Solanum virginianum $\mathrm{L}$. & Solanaceae & Undershrub & KRK \& RVS 106229 & Fruits \\
\hline
\end{tabular}




\begin{tabular}{|c|c|c|c|c|c|}
\hline S.No & Botanical Name & Family & Habit & Collection No. & Phenology \\
\hline 75 & Stephania japonica (Thunb.) Miers & Menispermaceae & Climber & KRK \& RVS 106211 & Flowers \& Fruits \\
\hline 76 & Streblus taxoides (B.Heyne ex Roth) Kurz & Moraceae & Shrub & RKM 106386 & Flowers \\
\hline 77 & Strychnos lenticellata Hill & Loganiaceae & Straggler & RKM 107506 & Vegetative \\
\hline 78 & Stylosanthes fruticosa (Retz.) Alston & Fabaceae & Herb & RKM 107534 & Flowers \\
\hline 79 & Syzygium alternifolium (Wight) Walp. & Myrtaceae & Tree & RKM 107521 & Vegetative \\
\hline 80 & Terminalia alata B.Heyne ex Roth & Combretaceae & Tree & RKM 106400 & Flowers \\
\hline 81 & Tylophora tetrapetala (Dennst.) Suresh & Asclepiadaceae & Twiner & RKM 107511 & Flowers \\
\hline 82 & Viscum ramosissimum Wall. ex Wight \& Arn. & Viscaceae & Parasitic herb & KRK \& RVS 106217 & Flowers \& Fruits \\
\hline 83 & Zehneria maysorensis (Wight \& Arn.) Arn. & Cucurbitaceae & Climber & KRK \& RVS 106319 & Flowers \& Fruits \\
\hline 84 & Ziziphus glabrata B.Heyne ex Roth & Rhamnaceae & Straggler & RKM 107505 & Fruits \\
\hline 85 & Ziziphus nummularia (Burm.f.) Wight \& Arn. & Rhamnaceae & Shrub & RKM 107502 & Flowers \\
\hline
\end{tabular}

RKM - R. Kottaimuthu; KRK - K. Ravikumar; RVS - R. Vijaya Sankar 